\title{
Homogeneous Two-Component Polycondensation without Strict Stoichiometric Balance via the Tsuji-Trost Reaction. Remote Control of Two Reaction Sites by Catalysis
}

\author{
Nobuyoshi Nomura ${ }^{1}$, Ko Tsurugi ${ }^{1}$, T. V. RajanBabu ${ }^{2}$ and Tadao Kondo ${ }^{1}$ \\ ${ }^{1}$ Laboratory of Polymer Chemistry, Graduate School of Bioagricultural Sciences, Nagoya \\ University, Nagoya 464-8601 Japan; ${ }^{2}$ Department of Chemistry, The Ohio State University, 100 \\ W. 18th Avenue, Columbus, Ohio 43210
}

\section{Experimental:}

All manipulations were conducted under a nitrogen atmosphere using standard Schlenk techniques. Nuclear magnetic resonance (NMR) spectra were recorded on a Varian JEMINI-2000 spectrometer for ${ }^{1} \mathrm{H}(300 \mathrm{MHz})$ and ${ }^{13} \mathrm{C}(75 \mathrm{MHz})$. IR spectra were obtained using a JASCO FT/IR-5MP spectrometer. Size exclusion chromatography (SEC) was conducted with a Tosoh SD-8020 system using Tosoh TSK-gel G2000H $\mathrm{HL}_{\mathrm{XL}}, \mathrm{G} 3000 \mathrm{H}_{\mathrm{XL}}$, and $\mathrm{G} 5000 \mathrm{H}_{\mathrm{XL}}$ columns in chloroform at $40{ }^{\circ} \mathrm{C}$ (polystyrene standards). Gel filtration was conducted using Sephadex LH-60 gel in $\mathrm{CHCl}_{3} / \mathrm{MeOH}(1 / 2)$ at $25^{\circ} \mathrm{C}$.

\section{Materials:}

$\mathrm{Pd}_{2}(\mathrm{dba})_{3}, \mathrm{dppb}$, and BSA were used as purchased from Aldrich, and BSA was stored under a nitrogen atmosphere. $\mathrm{PPh}_{3}$ was purchased from Kanto Chemical Co. Inc., Japan. Dry solvents such as toluene, diethyl ether, and tetrahydrofuran (THF) were distilled from $\mathrm{Na}$-benzophenone under $\mathrm{N}_{2}$. Dichloromethane, DMF, DMI, and HMPA were distilled from calcium hydride. Those solvents were stored under a nitrogen atmosphere.

\section{Remote Double Activation of Bifunctionality by Catalysis}

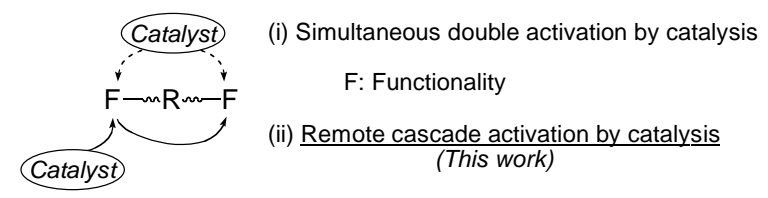

General procedure for polymerization (Table 1, entry 2):

The orange solution of $\mathrm{Pd}_{2}(\mathrm{dba})_{3}(4.7 \mathrm{mg}, 5.1 \mu \mathrm{mol})$, dppb (4.2 $\left.\mathrm{mg}, 9.8 \mu \mathrm{mol}\right)$, and BSA $(0.74 \mathrm{~mL}, 3.0 \mathrm{mmol})$ in $\mathrm{CH}_{2} \mathrm{Cl}_{2}(0.3 \mathrm{~mL})$ and DMF $(0.1 \mathrm{~mL})$ was stirred for $10 \mathrm{~min}$ at room temperature. To the solution of $2(276 \mathrm{mg}, 0.75 \mathrm{mmol})$ and $6(80 \mathrm{mg}, 0.50 \mathrm{mmol})$ in $\mathrm{CH}_{2} \mathrm{Cl}_{2}(0.6$ $\mathrm{mL}$ ), the orange solution of the palladium catalyst was transferred using a cannula, and the reaction was stirred at $25{ }^{\circ} \mathrm{C}$. Small amounts of crude samples from the reaction mixture were analyzed by ${ }^{1} \mathrm{H}$ NMR and SEC until the values of $M_{\mathrm{n}}$ reached the upper limits (2.5 days). At the completion of polymerization, $5 \mathrm{~mL}$ of $1 \mathrm{~N} \mathrm{HCl}$ was added to the reaction mixture, and the stirring was continued for $30 \mathrm{~min}$. The organic compounds were extracted with $\mathrm{CHCl}_{3}$ (ca. 20 $\mathrm{mL}$ ), and dried over $\mathrm{MgSO}_{4}$. After filtration, the filtrate was concentrated in vacuo, and the residue was purified by gel filtration to afford the analytically pure polymer $(102 \mathrm{mg}, 98 \%) . M_{\mathrm{n}}=$ $14,000, M_{\mathrm{w}} / M_{\mathrm{n}}=1.6_{2}$. 


\section{Scouting the electrophilic monomers for polycondensation out of stoichiometric control}

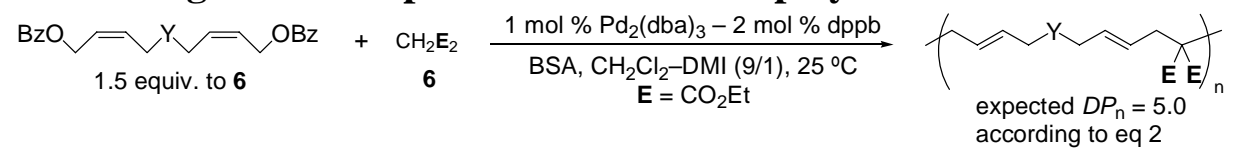

\begin{tabular}{ccccccc}
\hline Entry & $\mathrm{Y}$ & time, days & yield, $^{\mathrm{a}} \%$ & $M_{\mathrm{n}}{ }^{\mathrm{b}}$ & $M_{\mathrm{w}} / M_{\mathrm{n}}$ & obtained $D P_{\mathrm{n}}$ \\
\hline 1 & $\mathrm{CH}_{2}$ & $6.0(2.0)^{\mathrm{c}}$ & $-{ }^{\mathrm{d}}$ & $1,800(1,800)^{\mathrm{c}}$ & $1.4_{1}$ & $14(14)^{\mathrm{c}}$ \\
& & & & & $\left(1.4_{2}\right)^{\mathrm{c}}$ & \\
2 & $\mathrm{OCMe}_{2} \mathrm{O}$ & 1.0 & $-{ }^{\mathrm{d}}$ & 2,100 & $1.3_{3}$ & 7.6 \\
3 & $\mathrm{CHCO}_{2} \mathrm{Et}$ & 1.0 & $-{ }^{\mathrm{d}}$ & 1,800 & $1.3_{9}$ & 10 \\
4 & $\mathrm{O}$ & $6.0(2.5)^{\mathrm{c}}$ & 96 & $11,000(10,000)^{\mathrm{c}}$ & $1.6_{2}$ & $78(71)^{\mathrm{c}}$ \\
& & & & & $\left(1.6_{6}\right)^{\mathrm{c}}$ & \\
\hline
\end{tabular}

Reaction conditions: 6, 5.0 mmol; $\mathrm{Pd}_{2}(\mathrm{dba})_{3}, 5.0 \mu \mathrm{mol}$; dppb, $10 \mu \mathrm{mol}$; BSA, $3.0 \mathrm{mmol}$; solvent $\mathrm{CH}_{2} \mathrm{Cl}_{2}$-DMI (9/1); temp., $25^{\circ} \mathrm{C}$; Crude samples from the reaction mixture were analyzed every 6-12 h or 1-2 days until the values of $M_{\mathrm{n}}$ reached the upper limits.

${ }^{\mathrm{a}}$ Isolated yield. ${ }^{\mathrm{b}}$ Estimated by SEC (polystyene standards, $\mathrm{CHCl}_{3}$ ). ${ }^{\mathrm{c}}$ By analysis of crude samples of the polymerization mixture in the process of polycondensation. ${ }^{\mathrm{d}}$ Not isolated.

\section{Examination of the specific conditions for polycondensation out of stoichiometric control} using monomer $\underline{\mathbf{5}}$

\begin{tabular}{|c|c|c|c|c|}
\hline $\mathrm{BzO} \vee ح$ & $\begin{array}{r}\mathrm{Bz}_{2}+\mathrm{CH}_{2} \\
6\end{array}$ & $\begin{array}{c}1 \mathrm{~mol} \% \mathrm{Pd}_{2}(\mathrm{dba})_{3}-\text { ligand } \\
\mathrm{BSA}, \text { solvent, } 25 \text { 年 } \\
\mathrm{E}=\mathrm{CO}_{2} \mathrm{Et}\end{array}$ & \multicolumn{2}{|c|}{$\begin{array}{l}\text { expected } D P_{\mathrm{n}}=5.0 \\
\text { according to eq } 2\end{array}$} \\
\hline entry & ligand & solvent & $5 / 6$ & $\begin{array}{c}M_{\mathrm{n}}^{\mathrm{a}}(\text { obtained } \\
\left.D P_{\mathrm{n}}{ }^{\mathrm{a}}\right)\end{array}$ \\
\hline 1 & $\mathrm{dppb}$ & $\mathrm{CH}_{2} \mathrm{Cl}_{2}-\mathrm{DMI}(9 / 1)$ & $1.5 / 1.0$ & $11,000(78)$ \\
\hline 2 & $\mathrm{dppb}$ & $\mathrm{CH}_{2} \mathrm{Cl}_{2}-\mathrm{DMF}(9 / 1)$ & $1.5 / 1.0$ & $14,000(99)$ \\
\hline $3^{b}$ & $\mathrm{dppb}$ & $\mathrm{CH}_{2} \mathrm{Cl}_{2}-\mathrm{DMF}(9 / 1)$ & $1.0 / 1.5$ & $800(6)$ \\
\hline 4 & dppcyh $^{c}$ & $\mathrm{CH}_{2} \mathrm{Cl}_{2}-\mathrm{DMF}(9 / 1)$ & $1.5 / 1.0$ & $9,600(68)$ \\
\hline 5 & $2 \mathrm{PPh}_{3}$ & $\mathrm{CH}_{2} \mathrm{Cl}_{2}-\mathrm{DMF}(9 / 1)$ & $1.5 / 1.0$ & $-^{\mathrm{d}}$ \\
\hline 6 & $\mathrm{PPh}_{3}$ & $\mathrm{CH}_{2} \mathrm{Cl}_{2}-\mathrm{DMF}(9 / 1)$ & $1.5 / 1.0$ & $-{ }^{\mathrm{d}}$ \\
\hline 7 & $2 \mathrm{PCy}^{\mathrm{c}}$ & $\mathrm{CH}_{2} \mathrm{Cl}_{2}-\mathrm{DMF}(9 / 1)$ & $1.5 / 1.0$ & $-{ }^{\mathrm{d}}$ \\
\hline
\end{tabular}

All reactions were carried out with $0.50 \mathrm{mmol}$ of $6,3 \mathrm{mmol}$ of BSA, and $5 \mu \mathrm{mol}$ of $\mathrm{Pd}_{2}(\mathrm{dba})_{3}$ and $10 \mu \mathrm{mol}$ of the ligand (entries 1-4 and 6) or $20 \mu \mathrm{mol}$ of the ligand (entries 5 and 7) in $1 \mathrm{~mL}$ of the solvent under $\mathrm{N}_{2}$ at $25{ }^{\circ} \mathrm{C}$ unless otherwise noted. Reactions were monitored by SEC analysis of crude samples from the reaction mixture until $M_{\mathrm{n}}$ reached upper limits.

${ }^{\mathrm{a}}$ Estimated by SEC (polystyrene standards, $\left.\mathrm{CHCl}_{3}\right){ }^{\mathrm{b}}$ An excess amount of $\mathbf{6}$ was applied (2, 0.50 mmol; 6, $0.75 \mathrm{mmol}){ }^{c}$ See below. ${ }^{\mathrm{d}}$ Oligomers $\left(D P_{\mathrm{n}}<10\right)$ were formed.

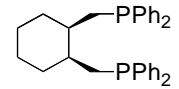

dppcyh

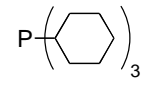

$\mathrm{PCy}_{3}$ 
Spectroscopic data of the polymers in Table 1:

$\operatorname{Poly}\{0 x y[(2 E, 7 E)-5,5-b i s(e t h o x y c a r b o n y l) n o n a-2,7-d i e n y l e n e]\}\left(\right.$ entries 1 and 2): ${ }^{1} \mathrm{H}$ NMR $\left(300 \mathrm{MHz}, \mathrm{CDCl}_{3}\right) \delta=1.24\left(\mathrm{t}, J=7.1 \mathrm{~Hz}, 3 \mathrm{H}, \mathrm{CO}_{2} \mathrm{CH}_{2} \mathrm{CH}_{3}\right), 2.61(\mathrm{~d}, J=6.6 \mathrm{~Hz}, 2 \mathrm{H}$, $\left.\mathrm{CCH}_{2} \mathrm{C}\right), 3.86\left(\mathrm{~d}, J=5.7 \mathrm{~Hz}, 2 \mathrm{H}, \mathrm{OCH}_{2} \mathrm{CH}=\mathrm{CH}\right), 4.17\left(\mathrm{q}, J=7.1 \mathrm{~Hz}, 2 \mathrm{H}, \mathrm{CO}_{2} \mathrm{CH}_{2}\right), 5.57(\mathrm{~m}, 2$ $\mathrm{H}, \mathrm{C}=\mathrm{CH}) ;{ }^{13} \mathrm{C} \mathrm{NMR}\left(75 \mathrm{MHz}, \mathrm{CDCl}_{3}\right) \delta=14.02\left(\mathrm{CO}_{2} \mathrm{CH}_{2} \mathrm{CH}_{3}\right), 35.28\left(\mathrm{CCH}_{2} \mathrm{C}\right), 57.40$ $\left(C\left(\mathrm{CO}_{2} \mathrm{Et}\right)_{2}\right), 61.22\left(\mathrm{CO}_{2} \mathrm{CH}_{2}\right), 70.06\left(\mathrm{OCH}_{2} \mathrm{CH}=\mathrm{CH}\right), 127.44,131.15$ (olefin), $170.68(C=\mathrm{O})$; IR $\left(\mathrm{KBr}, \mathrm{cm}^{-1}\right)$ 2981, 2937, 2857, 1730, 1445, 1367, 1263, 1200, 1097, 1042, 975, 859. Anal. Calcd. for $\mathrm{C}_{15} \mathrm{H}_{22} \mathrm{O}_{5}$ : C, 63.81; H, 7.85. Found: C, 63.79; H, 7.72.

$\operatorname{Poly}\{0 x y[(2 \boldsymbol{E}, 7 \boldsymbol{E})-5,5-d i a c e t o x y n o n a-2,7-d i e n y l e n e]\}$ (entry 4): ${ }^{1} \mathrm{H}$ NMR (300 MHz, $\left.\mathrm{CDCl}_{3}\right) \delta=2.10\left(\mathrm{~s}, 3 \mathrm{H}, \mathrm{C}(\mathrm{O}) \mathrm{CH}_{3}\right), 2.64\left(\mathrm{~d}, J=6.9 \mathrm{~Hz}, 2 \mathrm{H}, \mathrm{CCH}_{2} \mathrm{C}\right), 3.83(\mathrm{~d}, J=5.4 \mathrm{~Hz}, 2 \mathrm{H}$, $\left.\mathrm{OCH}_{2} \mathrm{CH}=\mathrm{CH}\right), 5.37\left(\mathrm{dt}, J=15.0 \mathrm{~Hz}, 7.5 \mathrm{~Hz}, 1 \mathrm{H}, \mathrm{OCH}_{2} \mathrm{CH}=\mathrm{CH}\right), 5.61(\mathrm{dt}, J=15.3 \mathrm{~Hz}, 6.0 \mathrm{~Hz}$, $\left.1 \mathrm{H}, \mathrm{OCH}_{2} \mathrm{CH}=\mathrm{CH}\right) ;{ }^{13} \mathrm{C} \mathrm{NMR}\left(75 \mathrm{MHz}, \mathrm{CDCl}_{3}\right) \delta=27.10\left(\mathrm{C}(\mathrm{O}) \mathrm{CH}_{3}\right), 33.44\left(\mathrm{CCH}_{2} \mathrm{C}\right), 69.84$, $70.27\left(\mathrm{OCH}_{2} \mathrm{CH}=\mathrm{CH}\right.$ and $\left.C\left(\mathrm{CO}_{2} \mathrm{Et}\right)_{2}\right), 127.02,131.17$ (olefin), $205.71(C=\mathrm{O})$; IR $\left(\mathrm{KBr}, \mathrm{cm}^{-1}\right)$ 2924, 2853, 1698, 1436, 1360, 1261, 1177, 1103, 1045, 973, 802.

$\operatorname{Poly}\{\operatorname{oxy}[(E)$-but-2-enylene $](1,3-i n d a n d i o n e-2,2-d i y l)[(E)$-but-2-enylene $]\}$ (entry 5): ${ }^{1} \mathrm{H}$ NMR $\left(300 \mathrm{MHz}, \mathrm{CDCl}_{3}\right) \delta=2.24\left(\mathrm{~d}, J=7.5 \mathrm{~Hz}, 2 \mathrm{H}, \mathrm{CCH}_{2} \mathrm{C}\right), 3.34(\mathrm{~d}, J=5.7 \mathrm{~Hz}, 2 \mathrm{H}$, $\left.\mathrm{OCH}_{2} \mathrm{CH}=\mathrm{CH}\right), 5.08\left(\mathrm{dt}, J=15.3 \mathrm{~Hz}, 7.5 \mathrm{~Hz}, 1 \mathrm{H}, \mathrm{OCH}_{2} \mathrm{CH}=\mathrm{CH}\right), 5.34(\mathrm{dt}, J=15.3 \mathrm{~Hz}, 6.2 \mathrm{~Hz}$, $\left.1 \mathrm{H}, \mathrm{OCH}_{2} \mathrm{CH}=\mathrm{CH}\right), 7.79-7.93(\mathrm{~m}, 2 \mathrm{H}, \mathrm{ArH}) ;{ }^{13} \mathrm{C} \mathrm{NMR}\left(75 \mathrm{MHz}, \mathrm{CDCl}_{3}\right) \delta=37.17\left(\mathrm{CCH}_{2} \mathrm{C}\right)$, $58.19\left(\mathrm{C}\left(\mathrm{CO}_{2} \mathrm{Et}\right)_{2}\right), 68.90\left(\mathrm{OCH}_{2} \mathrm{CH}=\mathrm{CH}\right), 123.11,126.33,131.41,135.86,142.13$ (benzene ring and olefin), $203.19(C=\mathrm{O})$; IR $\left(\mathrm{KBr}, \mathrm{cm}^{-1}\right) 3018,2912,2854,1742,1707,1595,1435,1357$, $1246,1106,1043,975,915,729$.

Poly\{oxy[(2E,12E)-5,5,10,10-tetrakis(ethoxycarbonyl)tetradeca-2,12-dien-7-ynylen e]\} (entry 6): ${ }^{1} \mathrm{H}$ NMR $\left(300 \mathrm{MHz}, \mathrm{CDCl}_{3}\right) \delta=1.24\left(\mathrm{t}, J=7.0 \mathrm{~Hz}, 6 \mathrm{H}, \mathrm{CH}_{3} \mathrm{CH}_{2} \mathrm{x}\right.$ 2), 2.72 (br, 4 $\left.\mathrm{H}, \mathrm{CH}_{2} \mathrm{C}\left(\mathrm{CO}_{2} \mathrm{Et}\right)_{2} \mathrm{CH}_{2}\right), 385\left(\mathrm{~d}, J=5.7 \mathrm{~Hz}, 2 \mathrm{H}, \mathrm{RCH}=\mathrm{CHCH}_{2} \mathrm{O}\right), 4.18(\mathrm{q}, J=7.1 \mathrm{~Hz}, 4 \mathrm{H}$, $\left.\mathrm{OCH}_{2} \mathrm{Me}\right), 5.47(\mathrm{dt}, \mathrm{J}=15.3,7.5 \mathrm{~Hz}, 1 \mathrm{H}, \mathrm{CH}=\mathrm{C}), 5.66(\mathrm{dt}, \mathrm{J}=15.0,5.9 \mathrm{~Hz}, \mathrm{CH}=\mathrm{C}) ;{ }^{13} \mathrm{C} \mathrm{NMR}$ $\left(75 \mathrm{MHz}, \mathrm{CDCl}_{3}\right) \delta=13.93\left(\mathrm{CH}_{3} \mathrm{CH}_{2} \mathrm{O}\right), 22.72\left(\mathrm{spC}-\mathrm{CH}_{2}\right), 34.76\left(\mathrm{sp}^{2} \mathrm{C}-\mathrm{CH}_{2} \mathrm{C}\left(\mathrm{CO}_{2} \mathrm{Et}\right)_{2}\right), 56.84$ $\left(C\left(\mathrm{CO}_{2} \mathrm{Et}\right)_{2}\right), 61.47\left(\mathrm{OCH}_{2} \mathrm{Me}\right), 70.01\left(\mathrm{C}=\mathrm{CCH}_{2} \mathrm{O}\right), 77.73(\mathrm{spC}), 126.91,131.58(C=C), 169.77$ $(C=\mathrm{O})$; IR $\left(\mathrm{KBr}, \mathrm{cm}^{-1}\right) 2981,2360,1733,1277,1203,1096,1067,859$.

Poly[(1E,3E)-6,6-bis(ethoxycarbonyl)hepta-1,3-dienylene] (major polymer of entries 7-9): ${ }^{1} \mathrm{H}$ NMR $\left(300 \mathrm{MHz}, \mathrm{CDCl}_{3}\right) \delta=1.22\left(\mathrm{t}, J=7.1 \mathrm{~Hz}, 3 \mathrm{H}, \mathrm{CO}_{2} \mathrm{CH}_{2} \mathrm{CH}_{3}\right), 2.59(\mathrm{~d}, J=6.9 \mathrm{~Hz}$, $\left.2 \mathrm{H}, \mathrm{CCH}_{2} \mathrm{C}\right), 4.16\left(\mathrm{q}, J=7.1 \mathrm{~Hz}, 2 \mathrm{H}, \mathrm{CO}_{2} \mathrm{CH}_{2}\right), 5.00-6.37(\mathrm{~m}, 2 \mathrm{H}, \mathrm{C}=\mathrm{CH}) ;{ }^{13} \mathrm{C} \mathrm{NMR}(75 \mathrm{MHz}$, $\left.\mathrm{CDCl}_{3}\right) \delta=14.05\left(\mathrm{CO}_{2} \mathrm{CH}_{2} \mathrm{CH}_{3}\right), 35.95\left(\mathrm{CCH}_{2} \mathrm{C}\right), 57.85\left(\mathrm{C}\left(\mathrm{CO}_{2} \mathrm{Et}\right)_{2}\right), 61.17\left(\mathrm{CO}_{2} \mathrm{CH}_{2}\right), 126.65$, 133.92 (olefin), $170.73(C=\mathrm{O})$; IR $\left(\mathrm{KBr}, \mathrm{cm}^{-1}\right) 2981,2937,1731,1445,1367,1284,1242,1186$, $1095,992,927,859,733$.

Poly\{(p-toluenesulfonylimino)[(2E,7E)-5,5-bis(ethoxycarbonyl)nona-2,7-dienylene] \} (entry 11): ${ }^{1} \mathrm{H}$ NMR $\left(300 \mathrm{MHz}, \mathrm{CDCl}_{3}\right) \delta=1.15\left(\mathrm{t}, J=7.2 \mathrm{~Hz}, 6 \mathrm{H}, \mathrm{CO}_{2} \mathrm{CH}_{2} \mathrm{CH}_{3}\right), 2.54(\mathrm{~d}, J=$ $\left.5.7 \mathrm{~Hz}, 4 \mathrm{H}, \mathrm{CCH}_{2} \mathrm{C}\right), 3.70\left(\mathrm{br}, 4 \mathrm{H}, \mathrm{NCH}_{2}\right), 4.08\left(\mathrm{q}, J=7.2 \mathrm{~Hz}, 4 \mathrm{H}, \mathrm{CO}_{2} \mathrm{CH}_{2}\right), 5.37(\mathrm{~m}, 4 \mathrm{H}$, $\left.\mathrm{C}=\mathrm{CHCH}_{2}\right), 6.56-7.17(\mathrm{~m}, 5 \mathrm{H}, \mathrm{ArH}) ;{ }^{13} \mathrm{C} \mathrm{NMR}\left(75 \mathrm{MHz}, \mathrm{CDCl}_{3}\right) \delta=13.93\left(\mathrm{CO}_{2} \mathrm{CH}_{2} \mathrm{CH}_{3}\right)$, $35.10\left(\mathrm{CCH}_{2} \mathrm{C}\right), 51.34\left(\mathrm{NCH}_{2}\right), 57.62\left(\mathrm{C}\left(\mathrm{CO}_{2} \mathrm{Et}\right)_{2}\right), 61.10\left(\mathrm{CO}_{2} \mathrm{CH}_{2}\right), 112.52,116.32,125.74$, 129.05, 130.38 (olefin and $C \mathrm{H}$ of benzene ring), 148.53 ( $\mathrm{NC}$ of benzene ring), $170.74(C=\mathrm{O})$; IR $\left(\mathrm{KBr}, \mathrm{cm}^{-1}\right)$ 2981, 1730, 1599, 1506, 1366, 1266, 1196, 1096, 1036, 973, 748, 692. 
Poly $\{1,2$-phenylene[ $(1 E, 6 E)-4,4-b i s($ ethoxycarbonyl)hepta-1,6-dienylene $]\} \quad$ (entry 12): ${ }^{1} \mathrm{H}$ NMR (300 MHz, $\left.\mathrm{CDCl}_{3}\right) \delta=1.14\left(\mathrm{t}, J=7.1 \mathrm{~Hz}, 3 \mathrm{H}, \mathrm{CO}_{2} \mathrm{CH}_{2} \mathrm{CH}_{3}\right), 2.76(\mathrm{~d}, J=6.9 \mathrm{~Hz}$, $\left.2 \mathrm{H}, \mathrm{CCH}_{2} \mathrm{C}\right), 4.10\left(\mathrm{q}, J=7.2 \mathrm{~Hz}, 2 \mathrm{H}, \mathrm{CO}_{2} \mathrm{CH}_{2}\right), 5.89(\mathrm{dt}, J=15.3 \mathrm{~Hz}, 7.7 \mathrm{~Hz}, 1 \mathrm{H}, \mathrm{ArCH}=\mathrm{CH})$, $6.60(\mathrm{~d}, J=15.3 \mathrm{~Hz}, 1 \mathrm{H}, \mathrm{ArCH}=\mathrm{CH}), 7.15-7.20(\mathrm{~m}, 1 \mathrm{H}, \mathrm{ArH}), 7.31-7.35(\mathrm{~m}, 1 \mathrm{H}, \mathrm{ArH}) ;{ }^{13} \mathrm{C}$ $\left.\operatorname{NMR}\left(75 \mathrm{MHz}, \mathrm{CDCl}_{3}\right) \delta=14.07\left(\mathrm{CO}_{2} \mathrm{CH}_{2} \mathrm{CH}_{3}\right), 36.74\left(\mathrm{CCH}_{2} \mathrm{C}\right), 58.19\left(\mathrm{C}_{\left(\mathrm{CO}_{2} \mathrm{Et}\right.}\right)_{2}\right), 61.28$ $\left(\mathrm{CO}_{2} \mathrm{CH}_{2}\right), 126.55,126.62,127.58,131.77,135.23$ (benzene ring and olefin), $170.74(\mathrm{C}=\mathrm{O})$; IR $\left(\mathrm{KBr}, \mathrm{cm}^{-1}\right)$ 2980, 1728, 1444, 1367, 1263, 1198, 1096, 1032, 966, 911, 860, 748. 